\title{
How to grade level of evidence of physical interventions for people with spinal cord injury?
}

\author{
Spinal Cord (2015) 53, 249; doi:10.1038/sc.2014.235; published online 23 December 2014
}

Evidence-based practice (EBP) is using the available scientific evidence in making clinical decisions. In the foundation of EBP lies the classification of level of evidence (LOE). This hierarchical classification in the field of medicine was first reported in the Canadian Task Force on Periodic Health Examination. ${ }^{1}$ It was later expanded by Sackett ${ }^{2}$ and thereafter on that point were several more classifications. Although LOE does not signify a good quality research, practice recommendations are commonly based on it. Systematic reviews of high-quality randomized controlled trials (RCTs) are considered to be the highest LOE and are generally given the highest grade of recommendation. In a recent article by Harvey et al., ${ }^{3}$ the adherence of 53 RCTs to the Consolidated Standards of Reporting Trials guidelines was found to be inadequate. It was quite surprising to see the low number of RCTs of physical interventions for people with spinal cord injury (SCI), published between 2003 and 2013. There is no uncertainty that there are numerous physical interventions available for people with SCI. Then what could be the possible reasons of such fewer RCTs of physical interventions? Could the low number of RCTs be attributed to the comparatively lower incidence of SCI or to variability in level of injury or to practical problems such as lack of standardized treatments and ethical constraints for the controls? It would be interesting to study the number of RCTs of physical interventions in other populations. The more important question to address would be, whether LOE classification needs to be different for physical interventions for people with SCI. Do we really need high quality and quantity of RCTs to answer critical questions about physical interventions for people with SCI, or is there a need to give study designs such as the cohort, case control and case series placed low in the hierarchy a better level of recommendation? With the present LOE classifications, it is likely that systematic reviews would be largely inconclusive, and there would be an overall poor level of evidence of physical interventions for people with SCI. Perhaps, there could be a need to develop a new level of evidence classification system for physical interventions for people with SCI.

\section{CONFLICT OF INTEREST}

The author declares no conflict of interest.

Akash Shah

Department of Physiotherapy, School of Allied Health Sciences, Manipal University, Manipal, India E-mail: akashshah89bpt@gmail.com

1 The periodic health examination. Canadian Task Force on the Periodic Health Examination. Can Med Assoc J 1979; 121: 1193-1254.

2 Sackett DL. Rules of evidence and clinical recommendations on the use of antithrombotic agents. Chest 1989; 95: 2S-4S.

3 Harvey LA, Glinsky JV, Bowden JL, Arora M. How well do randomised controlled trials of physical interventions for people with spinal cord injury adhere to the CONSORT guidelines? An analysis of trials published over a 10-year period. Spinal Cord 2014; 52: 795-802. 